\title{
Incorporating patients' views in planning services for women with HIV infection
}

\author{
P.D Kell, S E Barton, F C Boag
}

\begin{abstract}
Objectives-To determine the preferences of HIV seropositive women for out-patient care facilities.

Design-Cross sectional survey

Setting-An HIV out-patient clinic, a genitourinary medicine (GUM) clinic and a self support group for HIV seropositive women.

Subjects-Fifty consecutive HIV seropositive women attending the out-patient clinic and 18 women attending the self support group.

Results-Eighty percent of the women were comfortable in the HIV clinic. The discomfort felt by the remainder related to time spent in the waiting room. All the women reported feeling comfortable in the GUM clinic. However, $46 \%$ indicated a preference for a clinic attended only by female patients and $34 \%$ stated that they preferred only female stafi. At the time of the survey only $61 \%$ of women attending the self support group were attending an outpatient clinic. An HIV clinic with integrated family planning, gynaecology, social workers and a creche was a universal preference expressed by these women. They also indicated a preference for female doctors (83\%).

Conclusion-Women with HIV infection have clear views on the facilities which they would prefer to be available in regard to their out-patient care. In response to these wishes and particularly because of the high percentage of women not currently attending for outpatient care we have commenced a womens only clinic.
\end{abstract}

\section{Introduction}

By September 1991, 1848 women in the United Kingdom had been tested and found to be seropositive for antibodies to HIV-1. The true number is undoubtedly higher but the exact figure is unknown. Women therefore account for $11.4 \%$ of the known HIV-1 seropositive patients. ${ }^{1}$

The general development of services for people with HIV infection has been a complicated and sporadic process. In London, GUM clinics have provided the main focus for the provision of outpatient services for this group of patients. From this basis, local differences and initiatives for providing services for particular groups of patients have developed. These have been influenced by the apparent clinical demand, individual initiatives, the provision of research grants and support from charities.
The outcome of services based on such factors has often lacked co-ordination and audit of the services offered. Patients' preferences for care whilst attending GUM clinics have been studied $^{2} 3$ with conclusions identifying areas where changes can be made to increase patient satisfaction with the service. In order to plan the provision of clinical services for the increasing number of women presenting with HIV infection and to encourage women to attend for help and advice we felt it was important to evaluate the feelings and needs of the users of these services.

By December 1990, 120 women with HIV infection had attended this genito-urinary medicine clinic. The initial assessment is performed in the general GUM clinic (the John Hunter Clinic) but all patients are offered referral to the designated HIV unit (Kobler Centre) situated in the same building. The aim of our study was to assess HIV sero-positive women's views about the services which are currently being offered and those which they feel should be offered in the future.

\section{Methods}

Between September and December 1990, 50 consecutive HIV antibody women attending the John Hunter Clinic or Kobler Centre were given a self-administered questionnaire to complete. They could opt to complete the questionnaire anonymously if they so wished. A similar questionnaire was administered to 18 consecutive HIV antibody women attending a self-help group in London. In both questionnaires specific questions referred to the preference for the type of clinic the women wished to be seen in, the sex of the doctor, the facilites for children, an assessment of reasons for non-attendance and additional services they felt were needed.

\section{Results}

Fifty women with HIV infection attending our clinics received a questionnaire. No patients were unable or refused to complete the questionnaire. The results are in table 1 . The majority of women $(80 \%)$ felt comfortable being seen in the clinics in the Kobler Centre. Of the 10 women who said they felt uncomfortable, the specific reasons given identified time spent in the waiting room as the main cause for this feeling. Three women specifically stated they were distressed by seeing sick patients in the waiting room with them.

In contrast no patients expressed any feeling of discomfort while attending the 
Table 1 Views on 50 HIV antibody positive women attending our GUM and HIV out-patient clinics

\begin{tabular}{lc}
\hline & Numbers \\
\hline Discomfort felt attending GUM clinic & $0(0 \%)$ \\
Discomfort felt attending HIV clinic & $10(20 \%)$ \\
Preference for a female only clinic & $16(32 \%)$ \\
Preference for a female only HIV clinic & $23(46 \%)$ \\
Wanted a Social Worker, Health Adviser & $43(86 \%)$ \\
and Dietician to be available & $0(0 \%)$ \\
Requested creche facilities & \\
\hline
\end{tabular}

Table 2 HIV clinic facilties preferred by $18 \mathrm{HIV}$ antibody positive women attending a self-help group

\begin{tabular}{lc}
\hline & Numbers \\
\hline $\begin{array}{l}\text { Integrated Family Planning, Gynaecology } \\
\text { and Cervical Cytology }\end{array}$ & $18(100 \%)$ \\
Social Worker available & $18(100 \%)$ \\
Creche facilities & $18(100 \%)$ \\
A female doctor & $15(83 \%)$ \\
Counsellor available & $14(78 \%)$ \\
An integrated support group & $13(72 \%)$ \\
Female patients only & $9(50 \%)$ \\
All female staff & $6(33 \%)$ \\
\hline
\end{tabular}

genitourinary medicine clinic but 12 (24\%) specifically requested that smoking be allowed in the waiting rooms as they felt that a nosmoking policy contributed towards heightened anxiety. Two patients specifically stated that they felt that the music played in the waiting room was unhelpful in helping them relax. No women expressed the desire for a creche to be available. Women were asked whether they would favour the provision of a special clinic during which only female patients would be invited to attend. One third (22\%) felt that they would prefer to attend such a clinic. When asked if they would prefer this to be specifically for patients with HIV infection, $23(46 \%)$ felt that they would prefer to attend such a clinic. When asked which support services they thought would be useful to be permanently available within the clinics, more than $90 \%$ expressed the wish that Health Advisers, Social Workers, Counsellors and Dieticians be available during clinic hours.
Seventeen (34\%) respondents preferred that they had the option of women being available in these roles.

Eighteen consecutive HIV positive women attending a self-help group in London completed a different questionnaire assessing their views on health care. Only $11(61 \%)$ were currently attending clinics, and of these women four (29\%) felt that their requirements were being adequately met. When 18 were asked where they would choose to receive health care nine $(50 \%)$ specified a designated HIV clinic eight (44\%) a well woman's clinic and one $(5 \%)$ a GUM clinic. Table 2 delineates their specific requirements for out-patient care.

\section{Discussion}

This study describes specific requirements which women stated as desirable for their HIV out-patient care. These results from our clinic have an obvious inherent bias as some patients who were not happy being seen amongst predominantly male and/or homosexual patients may have "voted with their feet" and sought treatment elsewhere.

The women have expressed the need for additional on-site services such as availability of female doctors, gynaecology and family planning services. Furthermore, measures designed to reduce the distress of seeing people who have the same infection and are extremely unwell are clearly indicated. As upwards of one third of our patients would prefer to be seen in a women's only clinic, and such a service may encourage women not currently attending clinics to attend for health care, we commenced and are evaluating such a service.

1 Communicable Diseases Surveillance Centre/Communicable Diseases (Scotland) Unit Report. London. ISSN 0144-3186. 13 Dec 1991:223-4.

2 Munday PE. Genitourinary medicine services; consumers' views. Genitourin Med 1990;66:108-11.

3 Rogstad KE. Patients' assessment of and suggestions for a genitourinary medicine service. Int $\mathcal{f} S T D$ E $A I D S$ 1991;2:440-1. 